\title{
Herb-Induced Liver Injury Related to Reynoutria multiflora (Thunb.) Moldenke: Risk Factors, Molecular and Mechanistic Specifics
}

\author{
Xing-Ran Zhai ${ }^{1}$, Zheng-Sheng Zou ${ }^{1,2,3 *}$, Jia-Bo Wang ${ }^{3,4 *}$ and Xiao-He Xiao ${ }^{3,5}$ \\ ${ }^{1}$ Peking University 302 Clinical Medical School, Beijing, China, ${ }^{2}$ Medical School of Chinese PLA, Beijing, China, ${ }^{3}$ Senior \\ Department of Hepatology, the Fifth Medical Center of PLA General Hospital, Beijing, China, ${ }^{4}$ School of Traditional Chinese \\ Medicine, Capital Medical University, Beijing, China, ${ }^{5}$ China Military Institute of Chinese Medicine, the Fifth Medical Center, \\ Chinese PLA General Hospital, Beijing, China
}

OPEN ACCESS

Edited by:

Rong-Rong $\mathrm{He}$,

Jinan University, China

Reviewed by:

Lifeng Han,

Tianjin University of Traditional

Chinese Medicine, China

Xin $\mathrm{He}$,

Guangdong Pharmaceutical

University, China

*Correspondence:

Zheng-Sheng Zou

zszou302@163.com

Jia-Bo Wang

pharm_sci@126.com

Specialty section:

This article was submitted to

Ethnopharmacology,

a section of the journal

Frontiers in Pharmacology

Received: 09 July 2021

Accepted: 23 August 2021 Published: 02 September 2021

Citation:

Zhai X-R, Zou Z-S, Wang J-B and Xiao X-H (2021) Herb-Induced Liver Injury Related to Reynoutria multiflora

(Thunb.) Moldenke: Risk Factors,

Molecular and Mechanistic Specifics.

Front. Pharmacol. 12:738577.

doi: 10.3389/fphar.2021.738577
Herbal medicine is widely used in Asia as well as the west. Hepatotoxicity is one of the most severe side effects of herbal medicine which is an increasing concern around the world. Reynoutria multiflora (Thunb.) Moldenke (Polygonum multiflorum Thunb., PM) is the most common herb that can cause herb-induced liver injury (HILI). The recent scientific and technological advancements in clinical and basic research are paving the way for a better understanding of the molecular aspects of PM-related HILI (PM-HILI). This review provides an updated overview of the clinical characteristics, predisposing factors, hepatotoxic components, and molecular mechanisms of PM-HILI. It can also aid in a better understanding of HILI and help in further research on the same.

Keywords: drug-induced liver injury, herb-induced liver injury, predisposing factors, molecular mechanisms, Polygonum multiflorum Thunb., Reynoutria multiflora (Thunb.) Moldenke

\section{INTRODUCTION}

Herbal medicine, as a complementary and alternative medicine, is widely used in Asia as well as the west. Herbal medicine is generally considered to be natural, green, and harmless. In reality, herbal medicine may also have side effects, such as hepatotoxicity, nephrotoxicity, cardiotoxicity, neurotoxicity, and carcinogenicity. Hepatotoxicity is the most common side effect of great concern. The hepatotoxicity of drugs can be intrinsic or idiosyncratic (European Association for the Study of the Liver, 2019). The former refers to a liver injury that is closely related to drug dosage and timing, can be predicted, and has insignificant individual differences; the latter refers to a liver injury that is not correlated with the drug dosage and timing, is unpredictable, seen in a few people, and has a significant individual difference.

Herbal medicine is a common cause of drug-induced liver injury (DILI). A recent study carried out in Asian countries focusing on the causes of DILI reported that herbal medicine accounts for about 25\% of all DILI (Byeon et al., 2019; Shen et al., 2019). However, very few studies focus on herbinduced liver injury (HILI), and little is known about species, components, mechanisms, and predisposing factors of herbs that cause liver injury. Common herbs that can cause HILI include Reynoutria multiflora (Thunb.) Moldenke (Polygonum multiflorum Thunb., PM), Cullen corylifolium (L.) Medik., Corydalis yanhusuo (Y.H.Chou and Chun C. Hsu) W.T.Wang ex Z.Y.Su and C.Y.Wu, etc., of which the most common is PM, accounting for $32.3 \%$ of all cases (Byeon et al., 2019). PMrelated HILI (PM-HILI) is seen only in a few of the patients consuming this herb. 
TABLE 1 | Representative studies ${ }^{a}$ on liver injury caused by PM.

\begin{tabular}{|c|c|c|c|c|c|c|c|c|}
\hline $\begin{array}{l}\text { Number } \\
\text { of cases }\end{array}$ & Duration & $\begin{array}{l}\text { Male: } \\
\text { Female }\end{array}$ & $\begin{array}{l}\text { Age, } \\
\text { year }\end{array}$ & $\begin{array}{l}\text { Duration } \\
\text { of intake, } \\
\text { day }\end{array}$ & PM type & $\begin{array}{l}\text { Type } \\
\text { of liver } \\
\text { injury } \\
(\%)\end{array}$ & $\begin{array}{l}\text { Number } \\
\text { of deaths } \\
(\%)\end{array}$ & References \\
\hline 36 & 2000-2012 & 23: 13 & $24-73$ & $\begin{array}{c}<7(13.9 \%) ; 7-14(11.1 \%) ; 14-30 \\
(30.6 \%) ; 30-90(30.6 \%) ;>90(11.1 \%) ; \\
\text { Unknown (2.8\%) }\end{array}$ & $\begin{array}{l}\text { RPM } \\
\text { and } \\
\text { PPM }\end{array}$ & $\begin{array}{l}\text { Hepatocellular (58.3), } \\
\text { Cholestasis (5.6), } \\
\text { mixed (36.1) }\end{array}$ & $2(5.6)$ & $\begin{array}{l}\text { Zhang et al. (2013) } \\
\text { (Zhang et al., 2013b) }\end{array}$ \\
\hline 52 & 2006-2012 & 30: 22 & 22-69 & $5-120$ & PPM & $\begin{array}{l}\text { Hepatocellular (57.7), } \\
\text { Cholestasis (17.3), } \\
\text { mixed (25.0) }\end{array}$ & 0 & $\begin{array}{l}\text { Lian et al. (2013) (Lian } \\
\text { et al., 2013) }\end{array}$ \\
\hline 66 & 2009-2014 & 35: 31 & $8-63$ & $1-365$ & $\begin{array}{l}\text { RPM } \\
\text { and } \\
\text { PPM }\end{array}$ & $\begin{array}{l}\text { Hepatocellular (92.4), } \\
\text { Cholestasis (1.5), mixed (6.1) }\end{array}$ & $1(1.5)$ & $\begin{array}{l}\text { Zhu et al. (2015) (Zhu } \\
\text { et al., 2015) }\end{array}$ \\
\hline 60 & 2006-2010 & 28: 32 & $43.5^{b}$ & $11-168$ & RPM & $\begin{array}{l}\text { Hepatocellular (60.0), } \\
\text { Cholestasis (15.0), } \\
\text { mixed (25.0) }\end{array}$ & $1(1.7)$ & $\begin{array}{l}\text { Luo et al. (2016) (Luo } \\
\text { et al., 2016) }\end{array}$ \\
\hline 133 & 2012-2016 & $\begin{array}{l}\text { not } \\
\text { reported }\end{array}$ & $8-77$ & $5-224$ & $\begin{array}{l}\text { RPM } \\
\text { and } \\
\text { PPM }\end{array}$ & not reported & $\begin{array}{l}\text { not } \\
\text { reported }\end{array}$ & $\begin{array}{l}\text { Han et al. (2019) (Han } \\
\text { et al., 2019b) }\end{array}$ \\
\hline 140 & 2009-2018 & 60: 80 & $19-74$ & $1-150$ & $\begin{array}{l}\text { RPM } \\
\text { and } \\
\text { PPM }\end{array}$ & $\begin{array}{l}\text { Hepatocellular (94.3), } \\
\text { Cholestasis (2.9), mixed (2.9) }\end{array}$ & $1(0.71)$ & $\begin{array}{l}\text { Fu et al. (2020) (Fu } \\
\text { et al., 2020) }\end{array}$ \\
\hline
\end{tabular}

Abbreviations PM, Reynoutria multiflora (Thunb.) Moldenke; RPM, raw Reynoutria multiflora (Thunb.) Moldenke; PPM, processed Reynoutria multiflora (Thunb.) Moldenke.

a Studies with more than 30 cases are listed.

${ }^{b}$ Average age.

PM, the dried tuberous root of Reynoutria multiflora (Thunb.) Moldenke, when used as a traditional tonic and dietary supplement is believed to possess hypolipidemic, antiatherogenic, anti-aging, immunity-enhancing, neuroregulatory, hepatoprotective, and anti-inflammatory properties. These potentially beneficial effects contradict their role in liver injury. However, the potential beneficial effects and rare liver injury are common features of many herbs that cause liver injury. An updated review can help in better understanding the predisposing factors, hepatotoxic components, and molecular mechanisms of PM-HILI, and aid in its diagnosis, treatment, and prevention. It can also aid in a better understanding of HILI and help in further research on the same.

Although Polygonum multiflorum Thunb. is not an international standard Latin name, but it is widely used in scientific literature. In order to make it easier for readers to understand and relate to other literature, the abbreviation PM is used in this review.

\section{CLINICAL CHARACTERISTICS OF PM-HILI}

Reports of PM-HILI from China and other countries (But et al., 1996; Park et al., 2001; Mazzanti et al., 2004; Panis et al., 2005; Jung et al., 2011; Lian et al., 2013; Zhang Y. et al., 2013; Dong H. et al., 2014; Zhu et al., 2015; Luo et al., 2016; Li CY. et al., 2017; Han Z. X. et al., 2019; Fu et al., 2020) have increased in recent years (Table 1). Compared to other DILI, PM-HILI does not present specific clinical and pathological features. Hepatocellular injury is very common and cholestasis and mixed liver injury are rare. Common manifestations are jaundice and markedly increased levels of biochemical parameters such as total bilirubin, alanine aminotransferase, aspartate aminotransferase, etc. (Park et al., 2001; Mazzanti et al., 2004; Panis et al., 2005; Jung et al., 2011; Dong H. et al., 2014; Zhu et al., 2015; Li H. et al., 2017; Wang Y. et al., 2019). Most patients with PM-HILI have a good prognosis, although some patients may present with acute liver failure, chronic liver injury, or even death. PMHILI with underlying chronic liver disease, particularly alcoholic liver disease, has a worse prognosis and is more likely to develop into chronic DILI (Jing et al., 2019). Studies suggest that PM-HILI is an idiosyncratic drug-induced liver injury (IDILI). Clinical epidemiological analysis has found that PM-HILI only occurs in a very small number of individuals taking PM, which is a low-probability event (Zhang et al., 2020b). PM is usually prescribed at a wide range of doses over large periods in patients, which suggests the lack of a clear dose-dependent relationship (Zhu et al., 2015; Tu et al., 2019a; Zhang et al., 2020b). In clinical practice, $\mathrm{PM}$ has been prescribed at a dosage within the recommended range of Chinese Pharmacopeia (3-6 g/day for raw PM, 6-12 g/day for processed PM) in patients with PMHILI (Lei et al., 2015; Li CY. et al., 2017). PM-HILI is most commonly seen in patients with immune-related diseases such as vitiligo, psoriasis, rheumatoid arthritis, seborrheic alopecia, which are often accompanied by immune stress or mild elevation of inflammatory cytokines (Tu et al., 2019a), suggesting that immune stress may be an important predisposing factor for PM-HILI. Experimental animal studies have shown that only a long-term and large-dose administration of PM can exert hepatotoxic effects in healthy animals, while the corresponding dose is clinically difficult to achieve (Li et al., 2013; Zhu et al., 2014; Wang T. 
et al., 2015; Yang M. et al., 2016). However, PM can cause liver injury when administrated at a routine clinical dose within a short period in susceptible animal models (Li et al., 2015; Xie et al., 2016). Nevertheless, the phenomenon of high-dose and long-term administration of PM is also observed in clinical practice, and it cannot be ruled out that PM also exerts direct hepatotoxic effects when administered at very high doses.

\section{PREDISPOSING FACTORS AND BIOMARKERS}

\section{Mild Immune Stress}

Clinical analysis of PM-HILI cases has shown immune stress as one of the important predisposing factors for PM-HILI (Tu et al., 2019a). Tu et al. discovered that high-dose (50 g/kg) and longterm administration of PM to healthy rats did not cause obvious liver injury (Tu et al., 2015), but administration of PM with 2 times clinical-equivalent dose $(1.08 \mathrm{~g} / \mathrm{kg})$ to the MIS model rats induced by lipopolysaccharide (LPS) led to obvious liver injury, by which a model for evaluating the idiosyncratic hepatotoxicity of PM based on immune stress was established for the first time (Li et al., 2015). LPS is a major component of the cell wall of Gram-negative bacteria, which can activate the immune system and promote the release of pro-inflammatory cytokines, such as tumor necrosis factor (TNF)- $\alpha$, interleukin (IL)-1 $\beta$, IL-6, etc. A low dose of immune activator (such as LPS) can induce MIS, a mild and damage-free inflammatory response that stimulates immune stress or aberrant activation of immune cells of the body, thus increasing the susceptibility to PM-HILI.

Using multifactor detection, metabolomics approach, and receiver operating characteristic curve analysis, Tu et al. (Tu et al., 2019b) identified various susceptibility biomarkers for PM-HILI with MIS idiosyncratic hepatotoxic rat model, including 12 plasma chemokines or cytokines (IL-1 $\beta$, IL-6, IL-10, interferon (IFN) $-\gamma$, IFN- $\gamma$ induced protein (IP)-10, macrophage chemoattractant protein (MCP)-1, MIP-1a, MCP-3, growth-related oncogene protein- $\alpha$, granulocytemacrophage colony-stimulating factor, TNF- $\alpha$, Rantes) and 9 metabolites (L-Phenylalanine, Creatinine, L-Glutamine, N1, N5, N10-Tricoumaroyl spermidine, Dopamine, Acetone cyanohydrin, Glycocholic acid, Deethylatrazine, L-Valine).

A study that examined susceptibility biomarkers for PMHILI conducted by Zhang et al. (Zhang et al., 2020b) discovered significant differences in serum metabolites between susceptible and tolerant individuals. A total of 25 different metabolites were screened, involved in glycerol phospholipid, sphingolipids, fatty acid, histidine, and aromatic amino acid metabolism. A significant proportion of these metabolites are directly or indirectly involved in the regulation of immune and inflammatory responses. The serum levels of TNF- $\alpha$, IL- $1 \beta$, and IL- 6 were significantly increased in patients susceptible to PM-HILI compared with those tolerant to PM-HILI, with a threefold increase in TNF$a$, and its levels significantly correlated with multiple differential metabolites. It is recently reported that serum cytokine TNF- $\alpha$ and chemokine CCL2 or vascular endothelial growth factor (VEGF) as potential biomarkers of PM-HILI (Tu et al., 2021).

In short, when plasma and liver concentrations of inflammatory cytokines such as TNF- $\alpha$, IL-1 $\beta$, IL-6, MCP-1, VEGF are elevated, PM should be used with caution.

\section{Gene Polymorphism} Immune-Related Gene Polymorphism

In the last decade, numerous genome-wide association studies have linked human leukocyte antigens (HLAs) to IDILI susceptibility (Kaliyaperumal et al., 2018). Li et al. (Li et al., 2019) discovered that the frequency of $H L A-B \star 35: 01$ allele in patients with PM-HILI is approximately $45.4 \%$, significantly higher than in the general Han Chinese population $(2.7 \%)$. The incidence of PM-HILI in $H L A-B^{\star} 35: 01$ allele carriers is eight times higher than that in non-carriers. Therefore, it has been hypothesized that the $H L A-B^{\star} 35$ :01 allele is one of the susceptibility genes of PM-HILI in the Han Chinese population, and has potential value in predicting the occurrence of PM-HILI. A study by Yang et al. (Yang et al., 2020 ) showed the frequency of the $H L A-B^{\star} 35: 01$ allele is $41.1 \%$ in patients with PM-HILI, which is consistent with the above findings and confirms the association between $H L A-B^{\star} 35$ :01 allele and PM-HILI. The relationship between singlenucleotide polymorphisms (SNPs) and PM-HILI was investigated and rs1055348 in the HLA-B gene was identified as a potential susceptibility SNP specific to PMHILI. Rs1055348 has a high association with the $H L A-B^{\star} 35: 01$ allele and may serve as a tag for the $H L A-B^{\star} 35: 01$ allele. Rs 1055348 can be an alternative to $H L A-B{ }^{\star 35: 01}$ allele due to its low cost and easy detection. The above studies identified potential genetic susceptibility markers for PM-HILI, suggesting that screening for $H L A-B^{\star} 35: 01$ allele or tag SNP rs 1055348 may be beneficial in the identification of susceptible populations of PM-HILI in the Han Chinese population. It remains to be investigated whether $H L A-B^{\star} 35: 01$ or rs 1055348 has predictive value for susceptible individuals in non-Han Chinese populations.

\section{Metabolic Enzyme-Related Gene Polymorphism}

Cytochrome P-450 (CYP450) has polymorphisms. Alterations in allele frequency may lead to an increase or decrease in CYP450 enzyme activity, which is one of the reasons for individual differences in drug metabolism and drug response. Ma et al. (Ma et al., 2014) found that the frequency of $C Y P 1 A 2{ }^{\star} 1 C$ allele in patients with PM-HILI is $46.5 \%$, higher than that in healthy controls $(27.9 \%)$, suggesting CYP $1 A 2{ }^{*} 1 C$ mutation may cause PM-HILI. CYP1A2 is an important enzyme involved in the biotransformation of emodin ( $\mathrm{Ma}$ et al., 2014). CYP1A2*1C mutation is associated with decreased CYP1A2 activity (Zhou et al., 2009). The metabolism of emodin, an anthraquinone derivative in PM, may be inhibited, causing accumulation of emodin and promoting the progression of liver injury. It should be noted that the content of emodin in PM is low, and it may be difficult to reach a dose concentration clinically that 
A

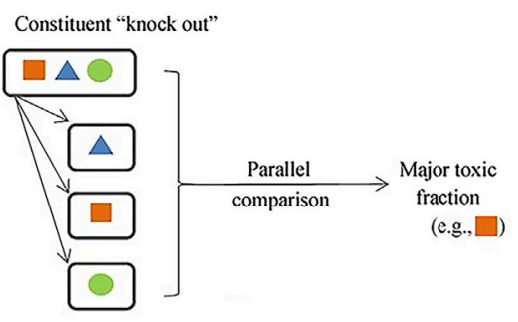

C

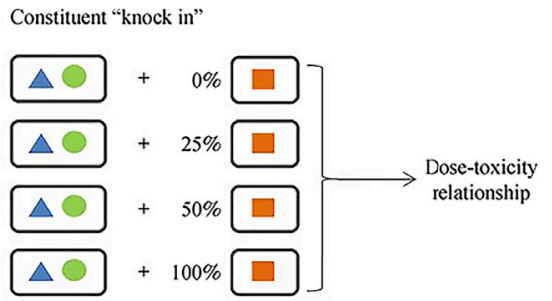

B

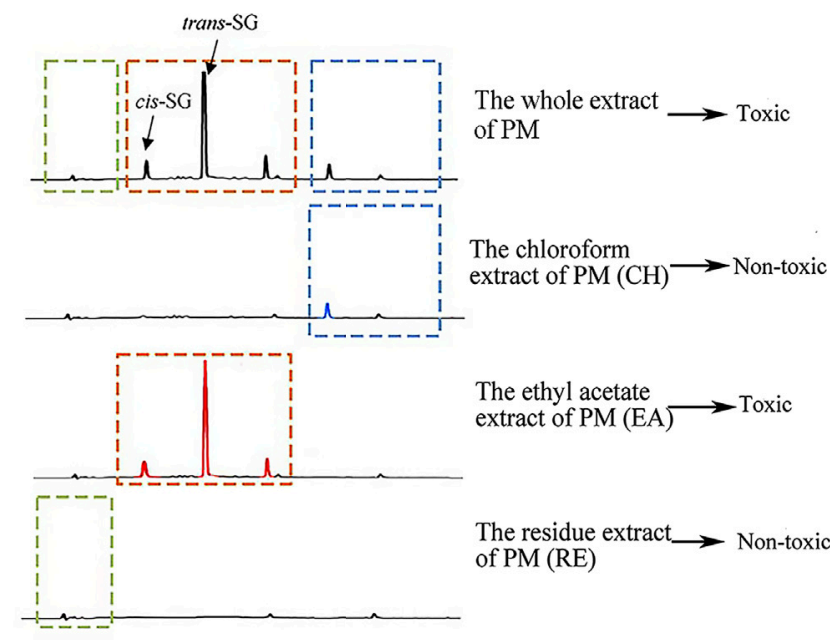

D

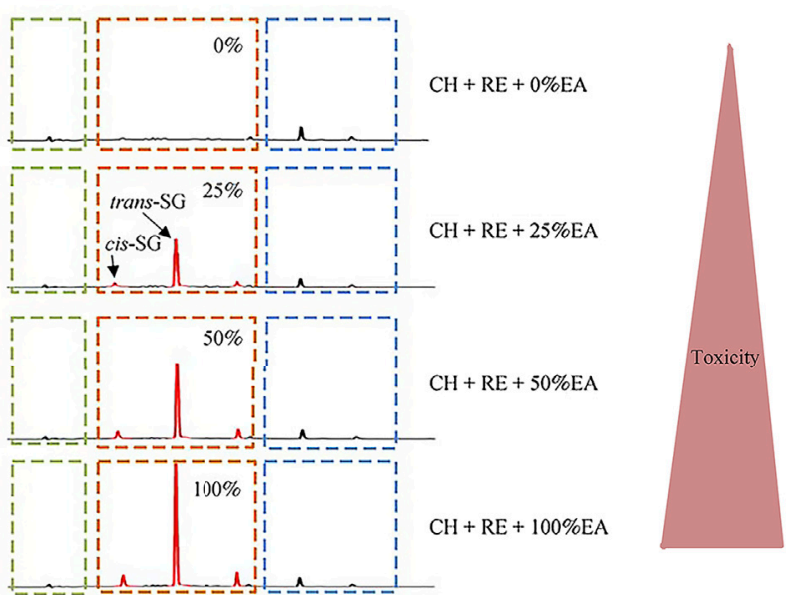

FIGURE 1 | Administration of the stilbene-containing ethyl acetate extract of PM to LPS-treated rats resulted in hepatotoxicity. Adapted from reference ( $L i \mathrm{C}$. et al., 2017), with permission from Springer, 2021. Abbreviations: trans-SG, trans-2,3,5,4'-tetrahydroxystilbene-2-O- $\beta$ - glucoside; cis-SG, cis-2,3,5,4'-tetrahydroxystilbene2-O- $\beta$-glucoside; PM, Reynoutria multiflora (Thunb.) Moldenke; $\mathrm{CH}$, the chloroform extract of PM; EA, the ethyl acetate extract of PM; RE, the residue extract of PM.

can cause liver injury. In contrast, consumption of herbs rich in emodin, such as rhubarb, causes much lesser liver injury than PM-HILI clinically. Currently, no animal and cell studies have confirmed the role of the $C Y P 1 A 2^{\star} 1 C$ allele in the occurrence of PM-HILI.

\section{Bile Acids}

A targeted metabolomics study by Dong et al. (Dong et al., 2015) showed that bile glycodeoxycholic acid levels and serum hyodeoxycholic acid (HDCA) levels are significantly decreased in PM-HILI rats, which may serve as potential biomarkers for PM-HILI. A study conducted by Zhao et al. (Zhao et al., 2017) showed that serum HDCA can be a potential biomarker for PM-HILI, and the result showed significantly increased serum HDCA in PM-HILI rats, in contradiction to the previous study. This may be due to the different dosages of PM administration and the different time points of HDCA measurement. A significant rise in Tauro$\beta$-muricholic acid levels in urine was observed, which may also be a potential biomarker for PM-HILI. These two biomarkers are positively correlated with the dosage and timing of PM and revert to the normal levels during the recovery period, suggesting good sensitivity. Exploring the sensitivity and specificity of biomarkers for PM-HILI is beneficial for accurate diagnosis and prompt commencement of therapy.

\section{MicroRNA-122}

It has been proposed that microRNA-122, a hepatocytespecific microRNA, is an ideal potential biomarker for liver disease (Fontana, 2014). Fan et al. (Fan et al., 2015) showed that serum microRNA-122 may also be a potential biomarker for PM-HILI. However, microRNA-122 may not be specific to PM-HILI, and its efficacy in clinical practice needs further testing. 


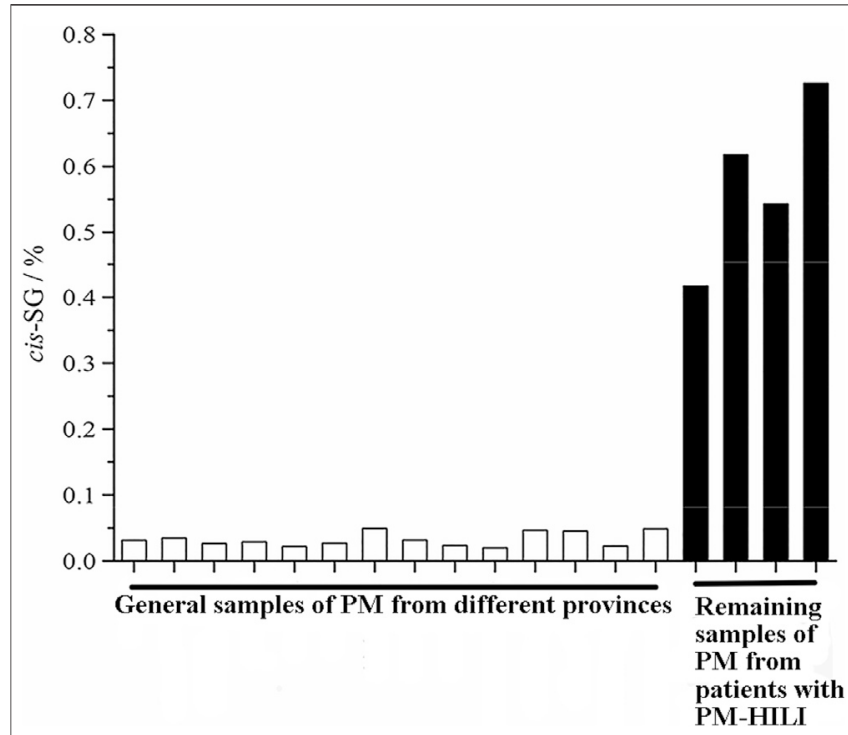

FIGURE 2 | The content of cis-SG in the remaining samples of PM from patients with PM-HILI is significantly higher than that of the general samples of PM from different provinces. Adapted with permission from reference (Zhang et al., 2017a). Abbreviations: cis-SG, cis-2,3,5,4'-tetrahydroxystilbene2-O- $\beta$-glucoside; PM, Reynoutria multiflora (Thunb.) Moldenke; PM-HILI, PMrelated herb-induced liver injury.

\section{IDIOSYNCRATIC HEPATOTOXICITY-RELATED COMPONENTS IN PM}

The identification of hepatotoxic components is important to reveal the hepatotoxic effect of herbal medicine. The chemical composition of PM is complex and includes stilbene glycosides, anthraquinones, tannins, flavonoids, phospholipids, micronutrients, etc. The current focus of literature reports has been on the hepatotoxic effects of stilbene glycosides and anthraquinones (Lin et al., 2015; Li et al., 2016; Zhang et al., 2017a; Lin et al., 2017; Zhang et al., 2018; Han L. et al., 2019; Lin Y. et al., 2019; He et al., 2019; Li H.-Y. et al., 2020; Zhang et al., 2020a; Li RL. et al., 2020; Liu et al., 2020).

Li et al. (Li C. et al., 2017) found that ethyl acetate (EA) extract is responsible for the idiosyncratic hepatotoxicity of PM using the "knock-out" and "knock-in" strategies (Figure 1). Using mass spectrometry, it has been identified that the EA extract of PM consists of trans-2,3,5,4'-tetrahydroxystilbene-2-O- $\beta$-glucoside (trans-SG), cis-2,3,5,4'-tetrahydroxystilbene-2-O- $\beta$-glucoside (cis-SG), and emodin-8-O-glucoside (EG). Li et al. (Li C. et al., 2017) used monomers and confirmed that cis-SG, and not transSG, is the important component responsible for the idiosyncratic hepatotoxicity in PM. Cis-SG shows a definite dose-toxicity relationship in the MIS model and the dose is nearly equivalent to the clinical dosage by weight. The content of cisSG in remaining samples of PM from patients with PM-HILI is significantly higher than that of general samples of PM from different provinces further proves that cis-SG is a hepatotoxicityrelated component of PM-HILI (Figure 2) (Zhang et al., 2017a).
The naturally occurring component in PM is largely trans-SG, and the content of cis-SG in PM is low. Trans-SG is the component responsible for beneficial effects and has properties of anti-oxidation (Tao et al., 2011; Zhang et al., 2012), antiinflammation (Zhang F. et al., 2013; Huang et al., 2013), antisenescence (Han et al., 2012; Büchter et al., 2015), and enhancing memory (Wang et al., 2011; Chen et al., 2016). Zhang et al. (Zhang et al., 2017a) studied the relationship between levels of cisSG and idiosyncratic liver injury of PM and found that the higher the cis-SG content, the greater the hepatotoxicity. They also showed that PM does not cause liver injury on the MIS model when the cis-SG content is less than $0.10 \%$, suggesting an upper safety limit for quality control of cis-SG in PM. Therefore, cis-SG is the component responsible for idiosyncratic hepatotoxicity in PM. However, ultraviolet irradiation can induce the transformation of trans-SG to cis-SG (Dong L. H. et al., 2014), resulting in the transformation of effective components into toxic components (Figure 3) and increasing the risk of liver injury. Therefore, exposure to light should be avoided during the preparation and storage of PM.

Zhang et al. (Zhang et al., 2020a) studied EG in the EA extract of PM and found that EG causes liver injury in the MIS rat model in a dose-dependent manner and the dose is nearly equivalent to the clinical dose by weight. It is indicated that EG is an important component in PM that is responsible for idiosyncratic hepatotoxicity. The upper safety limit of quality control of EG in PM is $0.17 \%$, determined using the method described earlier by Zhang et al. (China Association of Chinese Medicine, 2020).

Equimolar amounts of emodin do not cause liver injury, and the toxicity of combined anthraquinone is significantly higher than free anthraquinone in idiosyncratic hepatotoxicity, in contrast with the results of direct hepatotoxicity caused by the two compounds at very large doses (Yang M. et al., 2016). This helps in better understanding the fact that the toxicity of PM decreases after processing.

The finding of Ma et al. (Ma et al., 2015) and Gao et al. (Gao et al., 2017) showed that both the toxicity as well as the content of EG is reduced after processing (Figure 4), which indicates the hepatotoxicity of PM is highly correlated with EG. Reducing the idiosyncratic hepatotoxic components (or the susceptible components) of PM by processing is one of the important approaches to reduce toxicity.

Anthraquinones such as emodin (Lin et al., 2015; Zhang et al., 2018; Li H.-Y. et al., 2020), rhein (Li H.-Y. et al., 2020), and other components such as torachrysone-O-hexose (Han L. et al., 2019), dianthrones (Li H.-Y. et al., 2020), polygonnodes C1-C4 (Yang JB. et al., 2016), catechin (Li et al., 2016), etc. with intrinsic hepatotoxicity have also been investigated. Although the above components cause liver injury when administrated in large quantities, the quantity of the above components is very low in the clinical dosage of PM. Therefore, PM-HILI cannot be independently caused by the above components. However, they may play a secondary or synergistic role in the occurrence of PMHILI. Recognition of this may contribute to a more complete understanding of the complex multi-component mechanisms of PM-HILI. 


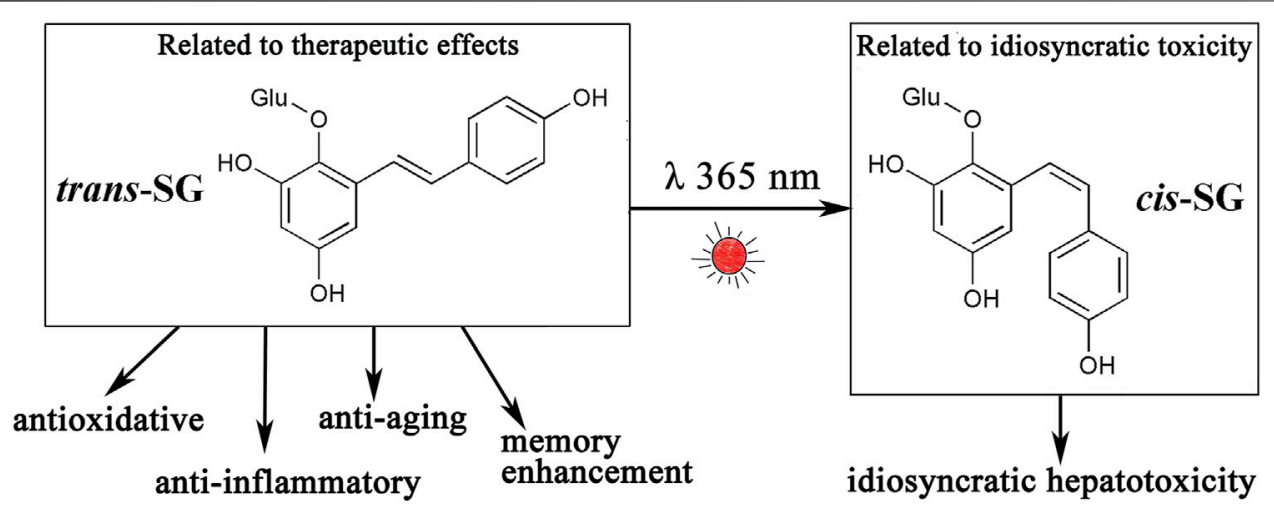

FIGURE 3 | Trans-SG can be transformed into its cis-isomer (cis-SG) by ultraviolet light or sunlight. Trans-SG is a component responsible for therapeutic effects, however, cis-SG is an idiosyncratic hepatotoxic component. Abbreviations: trans-SG, trans-2,3,5,4'-tetrahydroxystilbene-2-O- $\beta$ - glucoside; cis-SG, cis-2,3,5,4'tetrahydroxystilbene-2-O- $\beta$-glucoside.<smiles>Cc1cc(O)c2c(c1)C(=O)c1cc(O)cc(O)c1C2=O</smiles>

C<smiles>Cc1cc(O)c2c(c1)C(=O)c1cc(O)cc(O[C@@H]3O[C@H](CO)[C@@H](O)[C@H](O)[C@H]3O)c1C2=O</smiles>

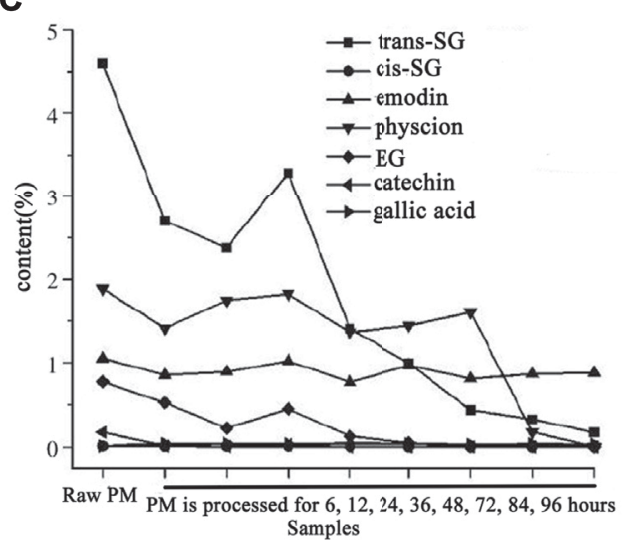

FIGURE 4 | The content of emodin-8-O-glucose (EG) of PM was reduced after processing. Adapted with permission from reference (Gao et al., 2017). Abbreviations: trans-SG, trans-2,3,5,4'-tetrahydroxystilbene-2-O- $\beta$-glucoside; cis-SG, cis-2,3,5,4'- tetrahydroxystilbene-2-O- $\beta$-glucoside; EG, emodin-8-O-glucoside; PM, Reynoutria multiflora (Thunb.) Moldenke.

\section{MOLECULAR MECHANISMS}

Current studies have shown that PM-HILI is considered to be IDILI and only occurs in a small number of susceptible individuals. However, in addition to IDILI, direct hepatotoxicity may be seen in specific PM-HILI patients who received a large dosage of PM. PM-HILI may be a result of a synergistic effect of multiple components and multiple mechanisms. Studies have shown that the molecular mechanism of PM-HILI is related to the inflammatory reaction, alterations of drug-metabolizing enzymes, apoptosis, and disorders of bile acid metabolism. A summary of the related 
mechanisms of PM-HILI around the two main susceptible components (cis-SG and EG) is presented below.

\section{Inflammatory Reaction}

Peroxisome proliferator-activated receptors (PPARs) belong to the nuclear receptor transcription factor superfamily. PPAR- $\gamma$, one subtype of PPARs, plays an important role in immune response and has a role in inhibiting the expression of inflammatory cytokines (Wu et al., 2020). Meng et al. (Meng et al., 2017) found that the mechanism of cis-SG in PM inducing idiosyncratic liver injury in the MIS rat model is associated with down-regulated expression of PPAR- $\gamma$, activation of the nuclear factor- $\kappa B$ signal pathway, secretion of proinflammatory cytokines such as TNF- $\alpha$ and IL- 6 by monocytes/macrophages; and the induction of hepatocyte apoptosis. Thus, the administration of a PPAR- $\gamma$ agonist (such as pioglitazone) in advance may prevent liver injury caused by cis-SG. Studies by Zhang et al. (Zhang et al., 2020a) and He et al. (He et al., 2017) have shown trans-SG in PM synergistically enhances the liver injury caused by LPS/EG and LPS/cis-SG and is associated with increased expression of proinflammatory cytokines and enhancement of inflammatory activity. Pan et al. (Pan et al., 2020) found that the activation of inflammasomes may also be a mechanism by which cis-SG induces IDILI. Previous studies have shown that MIS is a risk factor for PM-HILI. The pro-inflammatory effects of cis-SG and trans-SG and the involvement of inflammasomes prove that inflammation plays a role in PM-HILI.

\section{Alterations of Drug-Metabolizing Enzymes}

The up or down-regulation of drug-metabolizing enzymes may affect the metabolism of drugs and slow down the elimination of drugs from the body, and lead to toxic side effects. In recent years, studies have found that the chemical composition of PM affects the activity of drug-metabolizing enzymes.

Studies (Wang et al., 2015a; Wang M. X. et al., 2016) have found that emodin, an anthraquinone in $\mathrm{PM}$, has a role in inducing many CYP450 enzymes, such as CYP1A1, CYP1B1, CYP3A, CYP1A, CYP2B, CYP2E1, etc. Trans-SG has been found to increase the expression of CYP1A2 and CYP3A4 both in mice and in human normal liver L02 hepatocytes, and thus exacerbates the hepatotoxicity induced by acetaminophen in mice (Xu et al., 2017). Another study (Xing et al., 2019) also shows that trans-SG induces the activity of CYP1A2, and thus enhances the metabolism of emodin. Several components in PM have inhibitory effects on several uridine diphosphate glucuronosyltransferases (UGTs). EG, emodin-8-O-beta-Dglucoside, hydroxy emodin, emodin, cis-emodin dianthrones, trans-emodin dianthrones, and polygonumnolide C2 have a strong inhibitory effect on UGT1A1 (Wang Q. et al., 2016; Wang et al., 2017; Wang et al., 2018; Wang Q. et al., 2019); emodin plays a role in down-regulating the gene and protein expression of UGT2B7 (Wu et al., 2018); trans-SG has a role in down-regulating UGT1A8 levels (Ma et al., 2013). The effect of the above components on metabolic enzymes may be involved in the mechanisms of PM-HILI in susceptible individuals. However, no animal studies have demonstrated that alteration or knock-out of metabolic enzymes independently affects PM-HILI.

\section{Apoptosis}

Components related to toxicity in PM can promote the production of reactive oxygen species (ROS) and induce cell apoptosis, which may be the common endpoint of many mechanisms. A proteomic study (Lin L. et al., 2019) showed that emodin affects the oxidative phosphorylation pathway by inhibiting the function of mitochondrial respiratory chain complexes, which leads to an increase of ROS, the decrease of mitochondrial membrane potential, dysfunction of ATP synthesis, an increase in cytochrome $\mathrm{C}$ (Cyt C) release into the matrix, and the activation of the apoptotic factor asparticspecific caspase leading to mitochondrial energy metabolism disorder and hepatocyte apoptosis. Another study (Zhang et al., 2017b) found that the process by which emodin induces apoptosis may be mitochondrial cyclophilin D. Lai et al. (Lai et al., 2009) found that the ataxia-telangiectasia mutation (ATM) gene lies downstream of ROS and upstream of p53. Emodin leads to mitochondrial-dependent apoptosis by activating the ROS-ATMp53-Bax signaling pathway. Rhein was also reported (Bounda et al., 2015) to induce cell apoptosis via the mitochondrial pathway, and the specific mechanism is similar to the abovementioned process. Due to the low content of emodin in $\mathrm{PM}$, it may be difficult to obtain a hepatotoxic dosage causing liver injury clinically. However, the mild mitochondrial damage caused by low-dose emodin may result in the release of damageassociated molecular patterns, which may be concurrently involved in the pathogenesis of PM-HILI with other contributing risk factors.

\section{Disorders of Bile Acid Metabolism}

PM-HILI can be pathologically described as acute hepatitis, followed by cholestatic hepatitis (Wang Y. et al., 2019). Studies have shown that PM can cause bile acid metabolism disorders, and the mechanism may involve proteins and enzymes that affect the metabolism and transport of bile acid. Kang et al. (Kang et al., 2017) used sandwich-cultured rat hepatocytes to study the effects of anthraquinones such as emodin, chrysophanol, and physcion in PM on bile acid. Results showed that all three anthraquinones have a role in causing intracellular cholestasis. Of these, emodin inhibited the bile salt export pump (Bsep); chrysophanol inhibited the multidrug resistance-associated protein 2 , and all three anthraquinones inhibited the basolateral efflux transporters. Xue et al. (Wang X. et al., 2019) found that emodin inhibits Bsep by interfering with the interaction of 5 ' adenosine monophosphate-activated protein kinase and farnesoid X receptor (Fxr) in the liver, thereby playing a role in promoting intrahepatic cholestasis. Sun et al. (Sun et al., 2021) studied the effect of SG on bile acid and found significantly increased serum bile acid levels. SG inhibited Fxr and Bsep, leading to bile acid accumulation. SG inhibited 25hydroxycholesterol-7alpha-hydroxylase (CYP7B1) and interfered with the alternative pathway of bile acid synthesis.

Intrahepatic bile acid accumulation may have toxic effects on hepatocytes. On the one hand, bile acid disrupts the integrity of the mitochondrial membrane through its washing effect on lipids, leading to the production of ROS and release of Cyt C, resulting in the oxidative modification of lipids, proteins, and nucleic acids, 


\section{"firewood"}

Predisposing Factor

(Mild immune stress)

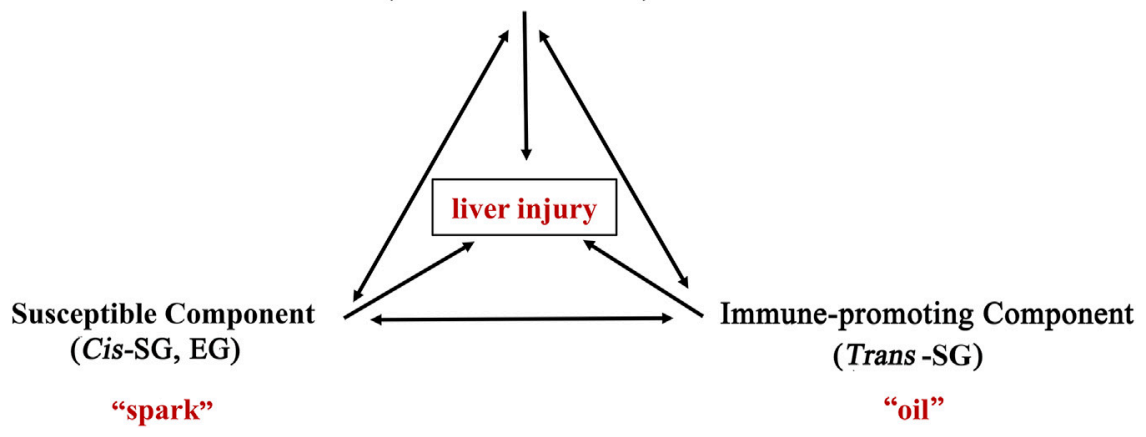

FIGURE 5 | The immunological stress-mediated tri-element injury hypothesis of idiosyncratic toxicity of PM. During an abnormally activated state (mild immune stress) of the body's immune system, the immune-promoting components in PM (trans-SG) may enhance the body's immune activation, and at the same time increase the susceptibility of the liver to certain components (cis-SG, EG), resulting in the overexpression of inflammatory cytokines, leading to liver injury. It is also called the "firewood-oil-spark hypothesis", in which the abnormally activated immune system is equivalent to "firewood", the immune-promoting components in PM are equivalent to "oil", and the potentially susceptible components for liver injury is equivalent to "spark". A single risk factor may not catch fire, but when three risk factors are present at the same time, a small spark may cause a raging fire (acute liver injury). Abbreviations: trans-SG, trans-2,3,5,4'-tetrahydroxystilbene-2-O- $\beta$-glucoside; cis-SG, cis- 2,3,5,4'-tetrahydroxystilbene-2-O- $\beta$-glucoside; EG, emodin-8-O-glucoside; PM, Reynoutria multiflora (Thunb.) Moldenke.

and ultimately mitochondrial-dependent apoptosis (Perez and Briz, 2009). On the other hand, bile acid causes endoplasmic reticulum stress and activates the programmed cell death pathway (Maillette de Buy Wenniger and Beuers, 2010). A causal relationship between increased bile acids and the occurrence of PM-HILI is unclear, but it cannot be ruled out that intrahepatic cholestasis may be a risk factor for PM-HILI.

\section{Others}

Emodin-glutathione (GSH) adducts (Qin et al., 2016; Jiang et al., 2017) can be formed by emodin or its metabolites of PM, and consequently, lead to hepatotoxicity. Emodin (Qin et al., 2016; Jiang et al., 2017) and aloe-emodin (Dong et al., 2017) have been reported to have a role in reducing GSH, an important intracellular antioxidant. GSH plays a role in maintaining the normal redox balance and protects the liver from oxidative stress. Reduction of GSH may lead to decreased ability to scavenge oxygen free radicals, resulting in oxidative stress response (Chen et al., 2009). However, the amounts of anthraquinones in PM may be too small to deplete GSH and cause liver injury, as the daily dose of even acetaminophen should be more than $4 \mathrm{~g}$ for GSH depletion to take place.

\section{HYPOTHESES OF THE MECHANISM OF PM-HILI}

Several hypotheses about the mechanism of IDILI have been established (Roth and Ganey, 2011; Bai et al., 2017), including inflammatory stress hypothesis, genetic polymorphism hypothesis, hapten hypothesis, mitochondrial dysfunction hypothesis, etc. Inflammatory stress hypothesis refers to that a state of inflammation stress of the body may increase the susceptibility of the liver to some drugs, resulting in IDILI. Several drugs of idiosyncratic nature like diclofenac (Deng et al., 2006), ranitidine (Luyendyk et al., 2003), chlorpromazine (Buchweitz et al., 2002), trovafloxacin (Shaw et al., 2007), etc. have been proven to induce IDILI in LPS models. HLAs- and metabolic enzyme-related gene polymorphism has been closely associated with IDILI. Studies have shown that $H L A-B^{\star 57: 01}$ (Monshi et al., 2013), $H L A-A^{\star} 33$ : 03 (Hirata et al., 2008), HLA-DQA1*02:01 (Spraggs et al., 2011), etc. are related to IDILI caused by flucloxacillin, ticlopidine, lapatinib, respectively. The gene polymorphism of UGT2B7, CYP2C8 (Daly et al., 2007), and CYP2E1 (Vuilleumier et al., 2006) has been reported to be related to IDILI caused by isoniazid. Hapten hypothesis refers to that some reactive drug metabolites bind to cellular proteins and act as haptens, which may induce an adaptive immune response (Uetrecht, 2007) and damage tissue by activating cells to release ROS, proteases, cytokines, etc. Studies have found that IDILI induced by multiple drugs including halothane (Bird and Williams, 1989), sulfamethoxazole (Callan et al., 2009), piperacillin (El-Ghaiesh et al., 2012), etc. is related to the hapten hypothesis. Multiple drugs (troglitazone, diclofenac, nimesulide, etc.) associated with IDILI exhibit a clear mitochondrial hazard (Boelsterli and Lim, 2007), which can lead to cell death. These hypotheses are derived from understanding IDILI from different perspectives or stages, and may not be mutually exclusive. IDILI is likely the result of multiple factors. Most of these hypotheses involve the immune system of the body, which plays a key role in the occurrence and development of IDILI.

Based on the inflammatory stress hypothesis, Rao et al. (Rao et al., 2020) considered PM-HILI to be an immune-mediated idiosyncratic liver injury. Further research has found that PM is more likely to cause liver injury in patients with immune 
activation. Also, individual components in PM have a potential role in promoting immune activation and causing liver injury. Wang et al. (Wang JB. et al., 2016; Wang J. B. et al., 2016) proposed the immune stress-mediated tri-element injury hypothesis of idiosyncratic toxicity of PM (Figure 5). It states that during an abnormally activated state (MIS) of the body's immune system, the immune-promoting components in PM (trans-SG) may enhance the body's immune activation, and at the same time increase the susceptibility of the liver to certain components (cis-SG, EG), resulting in the overexpression of inflammatory cytokines, leading to liver injury. It is also called the "firewood-oil-spark hypothesis", in which the abnormally activated immune system is equivalent to "firewood", the immune-promoting components in PM are equivalent to "oil", and the potentially susceptible components for liver injury is equivalent to "spark". A single risk factor may not catch fire, but when three risk factors are present at the same time, a small spark may cause a raging fire (acute liver injury). This hypothesis has been experimentally confirmed with animal models, providing new ideas for understanding PM-HILI. Indeed, this hypothesis systematically reveals the characteristics of the multi-component, multi-target effect, and immune synergy of the idiosyncratic HILI, providing new strategies for studying the mechanism of idiosyncratic HILI. In addition, it also suggests that PM should be avoided in combination with immune-promoting herbs and encouraged in combination with immune-suppressing herbs, which may be conducive to reduce liver injury. $H L A-B^{\star} 35: 01$ allele and rs1055348 may also be potentially involved in PMHILI, but evidence on the experimental animal models is not yet available and needs to be explored.

\section{REFERENCES}

Bai, Z. F., Gao, Y., Zuo, X. B., Wang, J. B., and Xiao, X. H. (2017). Progress in Research on the Pathogenesis of Immune Regulation and Idiosyncratic Drug-Induced Liver Injury. Acta Pharmaceutica Sinica 52 (07), 1019-1026.

Bird, G. L., and Williams, R. (1989). Detection of Antibodies to a Halothane Metabolite Hapten in Sera from Patients with Halothane-Associated Hepatitis. J. Hepatol. 9 (3), 366-373. doi:10.1016/0168-8278(89)90147-5

Boelsterli, U. A., and Lim, P. L. (2007). Mitochondrial Abnormalities-Aa Link to Idiosyncratic Drug Hepatotoxicity?. Toxicol. Appl. Pharmacol. 220 (1), 92-107. doi:10.1016/j.taap.2006.12.013

Bounda, G. A., Zhou, W., Wang, D. D., and Yu, F. (2015). Rhein Elicits In Vitro Cytotoxicity in Primary Human Liver HL-7702 Cells by Inducing Apoptosis through Mitochondria-Mediated Pathway. Evid. Based Complement. Alternat Med. 2015, 1-19. doi:10.1155/2015/329831

Büchter, C., Zhao, L., Havermann, S., Honnen, S., Fritz, G., Proksch, P., et al. (2015). TSG (2,3,5,4'-Tetrahydroxystilbene-2-O- $\beta$-D-Glucoside) from the Chinese Herb Polygonum Multiflorum Increases Life Span and Stress Resistance of Caenorhabditis elegans. Oxid Med. Cel Longev 2015, 124357. doi:10.1155/2015/124357

Buchweitz, J. P., Ganey, P. E., Bursian, S. J., and Roth, R. A. (2002). Underlying Endotoxemia Augments Toxic Responses to Chlorpromazine: Is There a Relationship to Drug Idiosyncrasy?. J. Pharmacol. Exp. Ther. 300 (2), 460-467. doi:10.1124/jpet.300.2.460

But, P. P., Tomlinson, B., and Lee, K. L. (1996). Hepatitis Related to the Chinese Medicine Shou-Wu-pian Manufactured from Polygonum Multiflorum. Vet. Hum. Toxicol. 38 (4), 280-282.

\section{PERSPECTIVES}

PM-HILI is a typical representative of HILI. Studying the risk factors, hepatotoxic components, and molecular mechanisms of PM-HILI is essential for a thorough understanding of the pathogenesis, treatment, and prevention of HILI. PM-HILI is considered to be IDILI and occurs only in a small number of susceptible individuals. The risk factors of PM-HILI mainly include MIS and gene polymorphism. The specific components responsible for the idiosyncratic hepatotoxicity are mainly cis-SG and EG. The processing technology of PM should be improved for quality control of cis-SG $(<0.10 \%)$ and EG $(<0.17 \%)$. Exposure to light should be avoided during the preparation and storage of PM for preventing the transformation from trans-SG to cis-SG. Underlying diseases, immune status, genetic background, etc. should be considered comprehensively when using PM and related preparations in clinical practice. The usage of biomarkers, such as $H L A-B^{\star} 35: 01$ allele, inflammatory cytokines like TNF- $\alpha$, MCP-1, VEGF, etc. to accurately identify the susceptible populations of PM-HILI is necessary for safe medication. PM should be encouraged in combination with immune-suppressing herbs for preventing liver injury. New mechanisms of PM-HILI need to be found and validated by in vivo and in vitro studies and complete the current hypothesis in the future.

\section{AUTHOR CONTRIBUTIONS}

XZ: writing original draft. JW, ZZ, and XX: review and editing.

Byeon, J. H., Kil, J. H., Ahn, Y. C., and Son, C. G. (2019). Systematic Review of Published Data on Herb Induced Liver Injury. J. Ethnopharmacol 233, 190-196. doi:10.1016/j.jep.2019.01.006

Callan, H. E., Jenkins, R. E., Maggs, J. L., Lavergne, S. N., Clarke, S. E., Naisbitt, D. J., et al. (2009). Multiple Adduction Reactions of Nitroso Sulfamethoxazole with Cysteinyl Residues of Peptides and Proteins: Implications for Hapten Formation. Chem. Res. Toxicol. 22 (5), 937-948. doi:10.1021/tx900034r

Chen, T., Yang, Y. J., Li, Y. K., Liu, J., Wu, P. F., Wang, F., et al. (2016). Chronic Administration Tetrahydroxystilbene Glucoside Promotes Hippocampal Memory and Synaptic Plasticity and Activates ERKs, CaMKII and SIRT1/ miR-134 In Vivo. J. Ethnopharmacol 190, 74-82. doi:10.1016/j.jep.2016.06.012

Chen, Y., Ji, L., Wang, H., and Wang, Z. (2009). Intracellular Glutathione Plays Important Roles in Pyrrolizidine Alkaloids-Induced Growth Inhibition on Hepatocytes. Environ. Toxicol. Pharmacol. 28 (3), 357-362. doi:10.1016/ j.etap.2009.06.002

China Association of Chinese Medicine (2020). Guidelines for Safe Use of Polygoni Multiflori Radix. Zhongguo Zhong Yao Za Zhi 45 (5), 961-966. doi:10.19540/ j.cnki.cjcmm.20191230.501

Daly, A. K., Aithal, G. P., Leathart, J. B., Swainsbury, R. A., Dang, T. S., and Day, C. P. (2007). Genetic Susceptibility to Diclofenac-Induced Hepatotoxicity: Contribution of UGT2B7, CYP2C8, and ABCC2 Genotypes. Gastroenterology 132 (1), 272-281. doi:10.1053/j.gastro.2006.11.023

Deng, X., Stachlewitz, R. F., Liguori, M. J., Blomme, E. A., Waring, J. F., Luyendyk, J. P., et al. (2006). Modest Inflammation Enhances Diclofenac Hepatotoxicity in Rats: Role of Neutrophils and Bacterial Translocation. J. Pharmacol. Exp. Ther. 319 (3), 1191-1199. doi:10.1124/jpet.106.110247

Dong, H., Slain, D., Cheng, J., Ma, W., and Liang, W. (2014a). Eighteen Cases of Liver Injury Following Ingestion of Polygonum Multiflorum. Complement. Ther. Med. 22 (1), 70-74. doi:10.1016/j.ctim.2013.12.008 
Dong, L. H., Guo, P. P., Guo, D., Yan, W. Y., and Wang, C. Y. (2014b). Preparation and Identification of Cis-2,3,5,4'-Tetrahydroxystilbene-2-O- $\beta$-D-Glucoside. J. Hebei Med. Univ. 35 (02), 166-169.

Dong, Q., Li, N., Li, Q., Zhang, C. E., Feng, W. W., Li, G. Q., et al. (2015). Screening for Biomarkers of Liver Injury Induced by Polygonum Multiflorum: a Targeted Metabolomic Study. Front. Pharmacol. 6, 217. doi:10.3389/fphar.2015.00217

Dong, X., Fu, J., Yin, X., Yang, C., and Ni, J. (2017). Aloe-emodin Induces Apoptosis in Human Liver HL-7702 Cells through Fas Death Pathway and the Mitochondrial Pathway by Generating Reactive Oxygen Species. Phytother Res. 31 (6), 927-936. doi:10.1002/ptr.5820

El-Ghaiesh, S., Monshi, M. M., Whitaker, P., Jenkins, R., Meng, X., Farrell, J., et al. (2012). Characterization of the Antigen Specificity of T-Cell Clones from Piperacillin-Hypersensitive Patients with Cystic Fibrosis. J. Pharmacol. Exp. Ther. 341 (3), 597-610. doi:10.1124/jpet.111.190900

European Association for the Study of the Liver (2019). EASL Clinical Practice Guidelines: Drug-Induced Liver Injury. J. Hepatol. 70 (6), 1222-1261. doi:10.1016/j.jhep.2019.02.014

Fan, X., Wang, J., Xie, L., Dong, Y., Han, G., Hu, D., et al. (2015). A New Animal Model for Polygonum Multiflorum Thunb-Induced Liver Injury in Rats and its Potential Mechanisms. Toxicol. Res. 4 (4), 1085-1097. doi:10.1039/c5tx00054h

Fontana, R. J. (2014). Pathogenesis of Idiosyncratic Drug-Induced Liver Injury and Clinical Perspectives. Gastroenterology 146 (4), 914-928. doi:10.1053/ j.gastro.2013.12.032

Fu, Q. B., Liu, T. C., Lei, Y., Lu, S., Fu, X., and Zhou, Z. (2020). Epidemiological and Clinical Characteristics of 140 Cases of Drug-Induced Liver Injury Caused by Polygonum Multiflorum and its Preparations. Chin. J. Integrated Traditional West. Med. Liver Dis. 30 (1), 6-9. doi:10.3969/j.issn.1005-0264.2020.01.002

Gao, D., Li, X. F., Yin, P., Wang, J. B., Sun, H. S., Li, F., et al. (2017). Preliminary Study on Hepatotoxic Components in Polygoni Multiflori Radix Based on Processing and Toxicity-Decreasing. Chin. Traditional Herbal Drugs 48 (10), 2044-2050.

Han, L., Wang, P., Wang, Y., Zhao, Q., Zheng, F., Dou, Z., et al. (2019a). Rapid Discovery of the Potential Toxic Compounds in Polygonum Multiflorum by UHPLC/Q-Orbitrap-MS-Based Metabolomics and Correlation Analysis. Front. Pharmacol. 10, 329. doi:10.3389/fphar.2019.00329

Han, X., Ling, S., Gan, W., Sun, L., Duan, J., and Xu, J. W. (2012). 2,3,5,4'tetrahydroxystilbene-2-O- $\beta$-d-glucoside Ameliorates Vascular Senescence and Improves Blood Flow Involving a Mechanism of P53 Deacetylation. Atherosclerosis 225 (1), 76-82. doi:10.1016/j.atherosclerosis.2012.08.011

Han, Z. X., Tu, C., Ge, F. L., Guo, Y. M., Jing, J., Zhang, L., et al. (2019b). Processing-related Differences of Polygoni Multiflori Radix and its Preparations-Induced Liver Injury Based on Reports. Chin. J. Pharmacoepidemiol. 28 (04), 227-232+258.

He, L., Yin, P., Meng, Y., Tang, J., He, T., Niu, M., et al. (2017). Immunological Synergistic Mechanisms of Trans-/cis-stilbene Glycosides in HeshouwuRelated Idiosyncratic Liver Injury. Sci. Bull. 62 (11), 748-751. doi:10.1016/ j.scib.2017.04.020

He, S., Zhang, X., Lu, S., Zhu, T., Sun, G., and Sun, X. (2019). A Computational Toxicology Approach to Screen the Hepatotoxic Ingredients in Traditional Chinese Medicines: Polygonum Multiflorum Thunb as a Case Study. Biomolecules 9 (10), 1-20. doi:10.3390/biom9100577

Hirata, K., Takagi, H., Yamamoto, M., Matsumoto, T., Nishiya, T., Mori, K., et al. (2008). Ticlopidine-induced Hepatotoxicity Is Associated with Specific Human Leukocyte Antigen Genomic Subtypes in Japanese Patients: a Preliminary CaseControl Study. Pharmacogenomics J. 8 (1), 29-33. doi:10.1038/sj.tpj.6500442

Huang, C., Wang, Y., Wang, J., Yao, W., Chen, X., and Zhang, W. (2013). TSG (2,3,4',5-tetrahydroxystilbene 2-O- $\beta$-D-Glucoside) Suppresses Induction of Pro-inflammatory Factors by Attenuating the Binding Activity of Nuclear Factor-Kb in Microglia. J. Neuroinflammation 10, 129. doi:10.1186/17422094-10-129

Jiang, L. L., Zhao, D. S., Fan, Y. X., Yu, Q., Li, P., and Li, H. J. (2017). Detection of Emodin Derived Glutathione Adduct in normal Rats Administered with Large Dosage of Polygoni Multiflori Radix. Front. Pharmacol. 8 (JUL), 446. doi:10.3389/fphar.2017.00446

Jing, J., Wang, R. L., Zhao, X. Y., Zhu, Y., Niu, M., Wang, L. F., et al. (2019). Association between the Concurrence of Pre-existing Chronic Liver Disease and Worse Prognosis in Patients with an Herb- Polygonum Multiflorum Thunb. Induced Liver Injury: a Case-Control Study from a Specialised Liver
Disease center in China. BMJ Open 9 (1), e023567. doi:10.1136/bmjopen-2018023567

Jung, K. A., Min, H. J., Yoo, S. S., Kim, H. J., Choi, S. N., Ha, C. Y., et al. (2011). Drug-Induced Liver Injury: Twenty Five Cases of Acute Hepatitis Following Ingestion of Polygonum Multiflorum Thunb. Gut Liver 5 (4), 493-499. doi:10.5009/gnl.2011.5.4.493

Kaliyaperumal, K., Grove, J. I., Delahay, R. M., Griffiths, W. J. H., Duckworth, A., and Aithal, G. P. (2018). Pharmacogenomics of Drug-Induced Liver Injury (DILI): Molecular Biology to Clinical Applications. J. Hepatol. 69 (4), 948-957. doi:10.1016/j.jhep.2018.05.013

Kang, L., Si, L., Rao, J., Li, D., Wu, Y., Wu, S., et al. (2017). Polygoni Multiflori Radix Derived Anthraquinones Alter Bile Acid Disposition in sandwichcultured Rat Hepatocytes. Toxicol. Vitro 40, 313-323. doi:10.1016/ j.tiv.2017.01.022

Lai, J. M., Chang, J. T., Wen, C. L., and Hsu, S. L. (2009). Emodin Induces a Reactive Oxygen Species-dependent and ATM-P53-Bax Mediated Cytotoxicity in Lung Cancer Cells. Eur. J. Pharmacol. 623 (1-3), 1-9. doi:10.1016/ j.ejphar.2009.08.031

Lei, X., Chen, J., Ren, J., Li, Y., Zhai, J., Mu, W., et al. (2015). Liver Damage Associated withPolygonum multiflorumThunb.: A Systematic Review of Case Reports and Case Series. Evid Based. Complement. Altern. Med., 2015, 1-9. doi:10.1155/2015/459749

Li, C., Niu, M., Bai, Z., Zhang, C., Zhao, Y., Li, R., et al. (2017a). Screening for Main Components Associated with the Idiosyncratic Hepatotoxicity of a Tonic Herb, Polygonum Multiflorum. Front. Med. 11 (2), 253-265. doi:10.1007/s11684-0170508-9

Li, C., Rao, T., Chen, X., Zou, Z., Wei, A., Tang, J., et al. (2019). HLA-B³5:01 Allele Is a Potential Biomarker for Predicting Polygonum Multiflorum-Induced Liver Injury in Humans. Hepatology 70 (1), 346-357. doi:10.1002/hep.30660

Li, C. Y., He, Q., Gao, D., Li, R. Y., Zhu, Y., Li, H. F., et al. (2017b). Idiosyncratic Drug-Induced Liver Injury Linked to Polygonum Multiflorum: A Case Study by Pharmacognosy. Chin. J. Integr. Med. 23 (8), 625-630. doi:10.1007/s11655-0172543-9

Li, C. Y., Li, X. F., Tu, C., Li, N., Ma, Z. J., Pang, J. Y., et al. (2015). The Idiosyncratic Hepatotoxicity of Polygonum Multiflorum Based on Endotoxin Model. Yao Xиe Xue Bao 50 (1), 28-33. doi:10.16438/j.0513-4870.2015.01.006

Li, H., Wang, X., Liu, Y., Pan, D., Wang, Y., Yang, N., et al. (2017c). Hepatoprotection and Hepatotoxicity of Heshouwu, a Chinese Medicinal Herb: Context of the Paradoxical Effect. Food Chem. Toxicol. 108, 407-418. doi:10.1016/j.fct.2016.07.035

Li, H.-Y., Yang, J.-B., Li, W.-F., Qiu, C.-X., Hu, G., Wang, S.-T., et al. (2020a). In Vivo hepatotoxicity Screening of Different Extracts, Components, and Constituents of Polygoni Multiflori Thunb. In Zebrafish (Danio rerio) Larvae. Biomed. Pharmacother. 131, 1-18. doi:10.1016/j.biopha.2020.110524

Li, Q., Zhao, K. J., Zhao, Y. L., Wang, J. B., Fang, F., Lv, Y., et al. (2013). High Dosage Administration of Polygonum Multiflorum Alcohol Extract Caused the Multi-Organ Injury in Rats. Glob. Traditional Chin. Med. 6 (01), 1-7.

Li, R. L., Gao, F., Yan, S. T., Ou, L., Li, M., Chen, L., et al. (2020b). Effects of Different Processed Products of Polygonum Multiflorum on the Liver. Evid. Based Complement. Alternat Med. 2020, 1-12. doi:10.1155/2020/5235271

Li, Y. M., Li, R. Y., Niu, M., Li, C. Y., Bai, Z. F., Feng, W. W., et al. (2016). Influence of Specification on Chemical Composition of Dissolution and Hepatocytes Toxicity of Polygonum Multiflorum. Zhongguo Zhong Yao Za Zhi 41 (6), 1033-1039. doi:10.4268/cjcmm20160610

Lian, X., Ke, T. T., and Hu, A. R. (2013). Clinical Analysis of 52 Patients with Liver Injury Induced by Polygonum Multiflorum and its Preparation. Chin. Arch. Traditional Chin. Med. 31 (05), 1133-1134.

Lin, L., Li, H., Lin, H., Zhang, M., Qu, C., Yan, L., et al. (2017). A New Perspective on Liver Injury by Traditional Chinese Herbs Such as Polygonum Multiflorum: The Geographical Area of Harvest as an Important Contributory Factor. Front. Pharmacol. 8, 349. doi:10.3389/fphar.2017.00349

Lin, L., Lin, H., Zhang, M., Ni, B., Yin, X., Qu, C., et al. (2015). A Novel Method to Analyze Hepatotoxic Components in Polygonum Multiflorum Using Ultraperformance Liquid Chromatography-Quadrupole Time-Of-Flight Mass Spectrometry. J. Hazard. Mater. 299, 249-259. doi:10.1016/j.jhazmat.2015.06.014

Lin, L., Liu, Y., Fu, S., Qu, C., Li, H., and Ni, J. (2019a). Inhibition of Mitochondrial Complex Function-The Hepatotoxicity Mechanism of Emodin Based on Quantitative Proteomic Analyses. Cells 8 (3), 263. doi:10.3390/cells8030263 
Lin, Y., Xiao, R., Yin, L. Z., Wu, P., Liu, Y. X., Li, C., et al. (2019b). Developing Spectrum-Toxicity Relationship with Rough Set Theory for Hepatotoxicity Material Basis of Polygonum Multiflorum. Zhongguo Zhong Yao Za Zhi 44 (3), 509-517. doi:10.19540/j.cnki.cjcmm.20181114.001

Liu, Y. L., Gao, D., Li, X. F., Xiao, X. H., and Wang, J. B. (2020). Preliminary Study on Hepatotoxic Components in Polygoni Multiflori Radix Based on Different Adjuvants Processing for Detoxification. Chin. Traditional Herbal Drugs 51 (2), 330-337. doi:10.7501/j.issn.0253-2670.2020.02.008

Luo, D. X., Wang, Z. Y., and Zhou, Z. (2016). Clinical Analysis of Liver Injury Caused by Raw Polygonum Multiflorum. J. Pract. Traditional Chin. Med. 32 (8), 836-837. doi:10.3969/j.issn.1004-2814.2016.08.076

Luyendyk, J. P., Maddox, J. F., Cosma, G. N., Ganey, P. E., Cockerell, G. L., and Roth, R. A. (2003). Ranitidine Treatment during a Modest Inflammatory Response Precipitates Idiosyncrasy-like Liver Injury in Rats. J. Pharmacol. Exp. Ther. 307 (1), 9-16. doi:10.1124/jpet.103.054288

Ma, J., Zheng, L., Deng, T., Li, C. L., He, Y. S., Li, H. J., et al. (2013). Stilbene Glucoside Inhibits the Glucuronidation of Emodin in Rats through the DownRegulation of UDP-Glucuronosyltransferases 1A8: Application to a Drug-Drug Interaction Study in Radix Polygoni Multiflori. J. Ethnopharmacol 147 (2), 335-340. doi:10.1016/j.jep.2013.03.013

Ma, K. F., Zhang, X. G., and Jia, H. Y. (2014). CYP1A2 Polymorphism in Chinese Patients with Acute Liver Injury Induced by Polygonum Multiflorum. Genet. Mol. Res. 13 (3), 5637-5643. doi:10.4238/2014.July.25.19

Ma, Z. J., Li, X. F., Lv, Y., Jiang, B. Q., Zhao, Y. L., Zhang, Y. M., et al. (2015). Comparative Study on Preparation of Polygoni Multiflori Radix Based on Hepatotoxic Bioassay. Zhongguo Zhong Yao Za Zhi 40 (12), 2325-2329. doi:10.4268/cjcmm20151212

Maillette de Buy Wenniger, L., and Beuers, U. (2010). Bile Salts and Cholestasis. Dig. Liver Dis. 42 (6), 409-418. doi:10.1016/j.dld.2010.03.015

Mazzanti, G., Battinelli, L., Daniele, C., Mastroianni, C. M., Lichtner, M., Coletta, S., et al. (2004). New Case of Acute Hepatitis Following the Consumption of Shou Wu Pian, a Chinese Herbal Product Derived from Polygonum Multiflorum. Ann. Intern. Med. 140 (7), W30. doi:10.7326/0003-4819-140-7200404060-00042-w3

Meng, Y. K., Li, C. Y., Li, R. Y., He, L. Z., Cui, H. R., Yin, P., et al. (2017). Cisstilbene Glucoside in Polygonum Multiflorum Induces Immunological Idiosyncratic Hepatotoxicity in LPS-Treated Rats by Suppressing PPAR- $\gamma$. Acta Pharmacol. Sin 38 (10), 1340-1352. doi:10.1038/aps.2017.32

Monshi, M. M., Faulkner, L., Gibson, A., Jenkins, R. E., Farrell, J., Earnshaw, C. J., et al. (2013). Human Leukocyte Antigen (HLA)-B ${ }^{\star 57: 01-r e s t r i c t e d ~ A c t i v a t i o n ~}$ of Drug-specific T Cells Provides the Immunological Basis for FlucloxacillinInduced Liver Injury. Hepatology 57 (2), 727-739. doi:10.1002/hep.26077

Pan, Y. Z., Li, Q. J., Zhang, Q., Jiang, B. P., Zhang, L., and Xu, L. (2020). The Compound Cell Model-Based Evaluation for Idiosyncratic Liver Injury of CisSG and Trans-SG. Acta Pharmaceutica Sinica 38, 1-17. doi:10.1038/aps.2017.32

Panis, B., Wong, D. R., Hooymans, P. M., De Smet, P. A., and Rosias, P. P. (2005). Recurrent Toxic Hepatitis in a Caucasian Girl Related to the Use of Shou-WuPian, a Chinese Herbal Preparation. J. Pediatr. Gastroenterol. Nutr. 41 (2), 256-258. doi:10.1097/01.mpg.0000164699.41282.67

Park, G. J., Mann, S. P., and Ngu, M. C. (2001). Acute Hepatitis Induced by Shou-WuPian, a Herbal Product Derived from Polygonum Multiflorum. J. Gastroenterol. Hepatol. 16 (1), 115-117. doi:10.1046/j.1440-1746.2001.02309.x

Perez, M. J., and Briz, O. (2009). Bile-acid-induced Cell Injury and protection. World J. Gastroenterol. 15 (14), 1677-1689. doi:10.3748/wjg.15.1677

Qin, B., Xu, Y., Chen, J., Huang, W., Peng, Y., and Zheng, J. (2016). Chemical Reactivity of Emodin and its Oxidative Metabolites to Thiols. Chem. Res. Toxicol. 29 (12), 2114-2124. doi:10.1021/acs.chemrestox.6b00191

Rao, T., Liu, Y. T., Zeng, X. C., Li, C. P., and Ou-Yang, D. S. (2020). The Hepatotoxicity of Polygonum Multiflorum: The Emerging Role of the Immune-Mediated Liver Injury. Acta Pharmacol. Sin 42, 27-35. doi:10.1038/ s41401-020-0360-3

Roth, R. A., and Ganey, P. E. (2011). Animal Models of Idiosyncratic Drug-Induced Liver Injury-Ccurrent Status. Crit. Rev. Toxicol. 41 (9), 723-739. doi:10.3109/ 10408444.2011.575765

Shaw, P. J., Hopfensperger, M. J., Ganey, P. E., and Roth, R. A. (2007). Lipopolysaccharide and Trovafloxacin Coexposure in Mice Causes Idiosyncrasy-like Liver Injury Dependent on Tumor Necrosis Factor-Alpha. Toxicol. Sci. 100 (1), 259-266. doi:10.1093/toxsci/kfm218
Shen, T., Liu, Y., Shang, J., Xie, Q., Li, J., Yan, M., et al. (2019). Incidence and Etiology of Drug-Induced Liver Injury in Mainland China. Gastroenterology 156 (8), 2230-e11. doi:10.1053/j.gastro.2019.02.002

Spraggs, C. F., Budde, L. R., Briley, L. P., Bing, N., Cox, C. J., King, K. S., et al. (2011). HLA-DQA1 ${ }^{\star 02: 01}$ Is a Major Risk Factor for Lapatinib-Induced Hepatotoxicity in Women with Advanced Breast Cancer. J. Clin. Oncol. 29 (6), 667-673. doi:10.1200/jco.2010.31.3197

Sun, M., Yu, Q. W., Xiang, T., Jiang, Z. Z., and Zhang, L. Y. (2021). 2,3,5,4'Tetrahydroxystibane-2-O- $\beta$-D-glucoside Induces Liver Injury by Disrupting Bile Acid Homeostasis and Phospholipids Efflux. Zhongguo Zhong Yao Za Zhi 46 (01), 139-145. doi:10.19540/j.cnki.cjcmm.20200818.401

Tao, L., Li, X., Zhang, L., Tian, J., Li, X., Sun, X., et al. (2011). Protective Effect of Tetrahydroxystilbene Glucoside on 6-OHDA-Induced Apoptosis in PC12 Cells through the ROS-NO Pathway. PLoS One 6 (10), e26055. doi:10.1371/ journal.pone. 0026055

Tu, C., He, Q., Li, C. Y., Niu, M., Han, Z. X., Ge, F. L., et al. (2019b). SusceptibilityRelated Factor and Biomarkers of Dietary Supplement Polygonum Multiflorum-Induced Liver Injury in Rats. Front. Pharmacol. 10, 335. doi:10.3389/fphar.2019.00335

Tu, C., Jiang, B. Q., Zhao, Y. L., Li, C. Y., Li, N., Li, X. F., et al. (2015). Comparison of Processed and Crude Polygoni Multiflori Radix Induced Rat Liver Injury and Screening for Sensitive Indicators. Zhongguo Zhong Yao Za Zhi 40 (4), 654-660. doi: $10.4268 / \mathrm{cjcmm} 20150416$

Tu, C., Niu, M., Wei, A. W., Tang, J. F., Zhang, L., Jing, J., et al. (2021), Susceptibility-Related Cytokine Panel for Prediction of Polygonum Multiflorum-Induced Hepatotoxicity in Humans. J. Inflamm. Res. 14, 645-655. doi:10.2147/jir.S299892

Tu, C., Ge, F. L., Guo, Y. M., Zhang, Y. M., Xiao, X. H., and Wang, J. B. (2019a). Analysis of Clinical Characteristics and Medication Rationality of Polygonum Multiflorum Thunb. And its Preparation-Related Liver Injury. Chin. J. Pharmacovigilance 16 (05), 270-276.

Uetrecht, J. (2007). Idiosyncratic Drug Reactions: Current Understanding. Annu. Rev. Pharmacol. Toxicol. 47, 513-539. doi:10.1146/ annurev.pharmtox.47.120505.105150

Vuilleumier, N., Rossier, M. F., Chiappe, A., Degoumois, F., Dayer, P., Mermillod, B., et al. (2006). CYP2E1 Genotype and Isoniazid-Induced Hepatotoxicity in Patients Treated for Latent Tuberculosis. Eur. J. Clin. Pharmacol. 62 (6), 423-429. doi:10.1007/s00228-006-0111-5

Wang, J. B., Cui, H. R., Bai, Z. F., and Xiao, X. H. (2016a). Precision MedicineOriented Safety Assessment Strategy for Traditional Chinese Medicines: Disease-Syndrome-Based Toxicology. Yao Xиe Xue Bao 51 (11), 1681-1688.

Wang, J. B., Li, C. Y., Zhu, Y., Song, H. B., Bai, Z. F., and Xiao, X. H. (2016b). Integrated Evidence Chain-Based Identification of Chinese Herbal MedicineInduced Hepatotoxicity and Rational Usage: Exemplification by Polygonum Multiflorum. Chin. Sci. Bull. 61 (09), 971-980.

Wang, M. X., Wang, Y. G., Xu, H. H., Zhang, Z. Y., Ma, Z. C., Xiao, C. R., et al. (2016c). Effects of Emodin in Polygonum Multiflorum on Liver Cytotoxicity and CYP450 Isoenzymes Expression in L02 Cells. Chin. Pharmacol. Bull. 32 (11), 1543-1548. doi:10.3969/j.issn.1001-1978.2016.11.013

Wang, Q., Wang, Y., Li, Y., Wen, B., Dai, Z., Ma, S., et al. (2017). Identification and Characterization of the Structure-Activity Relationships Involved in UGT1A1 Inhibition by Anthraquinone and Dianthrone Constituents of Polygonum Multiflorum. Sci. Rep. 7 (1), 17952. doi:10.1038/s41598-017-18231-y

Wang, Q., Dai, Z., Wang, Y. D., and Ma, S. C. (2018). Hepatotoxicity of Eight Components of Radix Polygonum Multiflorum Thunb. In Rat Liver Microsome System In Vitro. Chin. Pharm. J. 53 (8), 589-593. doi:10.11669/ cpj.2018.08.006

Wang, Q., Li, Y., Wang, Y. D., Wen, H. R., and Ma, S. C. (2019a). Investigation of Potential Hepatotoxic Components in Polygonum Multiflorum Based on Molecular Docking and Rat Liver Microsome Inhibition Test. Drug Eval. Res. 42 (04), 635-640.

Wang, Q. X., Lei, R. H., Wu, C. Q., Liao, M. Y., Xiao, X. H., and Wang, Q. J. (2015a). Induction of Cytochrome P450 Enzymes by Emodin in Liver Tissue of Rats. Drug Eval. Res. 38 (02), 147-150.

Wang, Q., Zhang, Y. J., Dai, Z., and Ma, S. C. (2016d). Study on the Hepatotoxicity of Monomers in Polygoni Multiflori Radix on the Basis of the Inhibition of Two-phase Metabolic Enzymes. Chin. J. Pharm. Anal. 36 (12), $2120-2124$. 
Wang, T., Wang, J. Y., Zhou, Z. X., Jiang, Z. Z., Li, Y. Y., Zhang, L., et al. (2015b). Study on Hepatotoxicity of Aqueous Extracts of Polygonum Multiflorum in Rats after 28-day Oral Administration: Cholestasis-Related Mechanism. Zhongguo Zhong Yao Za Zhi 40 (11), 2163-2167. doi:10.4268/cjcmm20151118

Wang, T., Yang, Y. J., Wu, P. F., Wang, W., Hu, Z. L., Long, L. H., et al. (2011). Tetrahydroxystilbene Glucoside, a Plant-Derived Cognitive Enhancer, Promotes Hippocampal Synaptic Plasticity. Eur. J. Pharmacol. 650 (1), 206-214. doi:10.1016/j.ejphar.2010.10.002

Wang, X., Han, L., Bi, Y., Li, C., Gao, X., Fan, G., et al. (2019b). Paradoxical Effects of Emodin on ANIT-Induced Intrahepatic Cholestasis and Herb-Induced Hepatotoxicity in Mice. Toxicol. Sci. 168 (1), 264-278. doi:10.1093/toxsci/ kfy295

Wang, Y., Wang, L., Saxena, R., Wee, A., Yang, R., Tian, Q., et al. (2019c). Clinicopathological Features of $\mathrm{He}$ Shou Wu-induced Liver Injury: This Ancient Anti-aging Therapy Is Not Liver-Friendly. Liver Int. 39 (2), 389-400. doi:10.1111/liv.13939

Wu, L., Guo, C., and Wu, J. (2020). Therapeutic Potential of PPAR $\gamma$ Natural Agonists in Liver Diseases. J. Cel Mol Med 24 (5), 2736-2748. doi:10.1111/ jcmm.15028

Wu, L., Han, W., Chen, Y., Zhang, T., Liu, J., Zhong, S., et al. (2018). Gender Differences in the Hepatotoxicity and Toxicokinetics of Emodin: The Potential Mechanisms Mediated by UGT2B7 and MRP2. Mol. Pharm. 15 (9), 3931-3945. doi:10.1021/acs.molpharmaceut.8b00387

Xie, L. H., Fan, X., Wu, C. Q., Jing, X., Li, M., Dong, Y. S., et al. (2016). Effects of Ethanol Extract from Polygonum Multiflorum on CYP450s during LPS Activated in Rats. Drug Eval. Res. 39 (02), 175-182.

Xing, Y., Wang, L., Wang, C., Zhang, Y., Zhang, Y., Hu, L., et al. (2019). Pharmacokinetic Studies Unveiled the Drug-Drug Interaction between Trans-2,3,5,4'-tetrahydroxystilbene-2-O- $\beta$-d-glucopyranoside and Emodin that May Contribute to the Idiosyncratic Hepatotoxicity of Polygoni Multiflori Radix. J. Pharm. Biomed. Anal. 164, 672-680. doi:10.1016/ j.jpba.2018.11.034

Xu, S., Liu, J., Shi, J., Wang, Z., and Ji, L. (2017). 2,3,4',5-tetrahydroxystilbene-2-O$\beta$-D-glucoside Exacerbates Acetaminophen-Induced Hepatotoxicity by Inducing Hepatic Expression of CYP2E1, CYP3A4 and CYP1A2. Sci. Rep. 7 (1), 16511. doi:10.1038/s41598-017-16688-5

Yang, J. B., Li, L., Dai, Z., Wu, Y., Geng, X. C., Li, B., et al. (2016a). Polygonumnolides C1-C4; Minor Dianthrone Glycosides from the Roots of Polygonum Multiflorum Thunb. J. Asian Nat. Prod. Res. 18 (9), 813-822. doi:10.1080/10286020.2016.1171758

Yang, M., Liu, T., Feng, W. H., Hui, L. Q., Li, R. R., Liu, X. Q., et al. (2016b). Exploration Research on Hepatotoxic Constituents from Polygonum Multiflorum Root. Zhongguo Zhong Yao Za Zhi 41 (7), 1289-1296. doi:10.4268/cjcmm20160721

Yang, W. N., Pang, L. L., Zhou, J. Y., Qiu, Y. W., Miao, L., Wang, S. Y., et al. (2020). Single-nucleotide Polymorphisms of HLA and Polygonum MultiflorumInduced Liver Injury in the Han Chinese Population. World J. Gastroenterol. 26 (12), 1329-1339. doi:10.3748/wjg.v26.i12.1329

Zhang, F., Wang, Y. Y., Yang, J., Lu, Y. F., Liu, J., and Shi, J. S. (2013a). Tetrahydroxystilbene Glucoside Attenuates Neuroinflammation through the Inhibition of Microglia Activation. Oxid Med. Cel Longev 2013, 680545. doi:10.1155/2013/680545

Zhang, J. K., Yang, L., Meng, G. L., Fan, J., Chen, J. Z., He, Q. Z., et al. (2012). Protective Effect of Tetrahydroxystilbene Glucoside against Hydrogen Peroxide-Induced Dysfunction and Oxidative Stress in Osteoblastic MC3T3E1 Cells. Eur. J. Pharmacol. 689 (1-3), 31-37. doi:10.1016/j.ejphar.2012.05.045
Zhang, L., He, D., Li, K., Liu, H., Wang, B., Zheng, L., et al. (2017b). Emodin Targets Mitochondrial Cyclophilin D to Induce Apoptosis in HepG2 Cells. Biomed. Pharmacother. 90, 222-228. doi:10.1016/j.biopha.2017.03.046

Zhang, L., Liu, X., Tu, C., Li, C., Song, D., Zhu, J., et al. (2020a). Components Synergy between Stilbenes and Emodin Derivatives Contributes to Hepatotoxicity Induced by Polygonum Multiflorum. Xenobiotica 50 (5), 515-525. doi:10.1080/00498254.2019.1658138

Zhang, L., Niu, M., Wei, A. W., Tang, J. F., Tu, C., Bai, Z. F., et al. (2020b). Risk Profiling Using Metabolomic Characteristics for Susceptible Individuals of Drug-Induced Liver Injury Caused by Polygonum Multiflorum. Arch. Toxicol. 94 (1), 245-256. doi:10.1007/s00204-019-02595-3

Zhang, L., Bai, Z. F., Li, C. Y., Hu, H. W. Y., Sha, M. C., Liu, Z. X., et al. (2017a). Study on Idiosyncratic Liver Injury and Content of Cis-2,3,5,4'-TetrahyDroxystilbene-2-O- $\beta$-D-Glucoside in Radix Polygoni Multiflori Preparata. Acta Pharmaceutica Sinica 52 (7), 1041-1047. doi:10.16438/j.05134870.2017-0307

Zhang, M., Lin, L., Lin, H., Qu, C., Yan, L., and Ni, J. (2018). Interpretation the Hepatotoxicity Based on Pharmacokinetics Investigated through Oral Administrated Different Extraction Parts of Polygonum Multiflorum on Rats. Front. Pharmacol. 9, 505. doi:10.3389/fphar.2018.00505

Zhang, Y., Cheng, S., and Lu, L. (2013b). Clinical Analysis of 36 Cases of DrugInduced Liver Injury by Polygonum Multiflorum Thunb. Hainan Med. J. 24 (02), 235-237.

Zhao, D. S., Jiang, L. L., Fan, Y. X., Dong, L. C., Ma, J., Dong, X., et al. (2017). Identification of Urine Tauro- $\beta$-Muricholic Acid as a Promising Biomarker in Polygoni Multiflori Radix-Induced Hepatotoxicity by Targeted Metabolomics of Bile Acids. Food Chem. Toxicol. 108, 532-542. doi:10.1016/j.fct.2017.02.030

Zhou, S. F., Yang, L. P., Zhou, Z. W., Liu, Y. H., and Chan, E. (2009). Insights into the Substrate Specificity, Inhibitors, Regulation, and Polymorphisms and the Clinical Impact of Human Cytochrome P450 1A2. Aaps j 11 (3), 481-494. doi:10.1208/s12248-009-9127-y

Zhu, X. G., Zhang, S. Y., Jiang, W., Zhang, G. P., Li, J. S., Yang, H. R., et al. (2014). Study on the Long-Term Toxicity and Safety Pharmacological Effects of Raw Polygonum Multiflorum. J. Toxicol. 28 (06), 483-485.

Zhu, Y., Liu, S. H., Wang, J. B., Song, H. B., Li, Y. G., He, T. T., et al. (2015). Clinical Analysis of Drug-Induced Liver Injury Caused by Polygonum Multiflorum and its Preparations. Zhongguo Zhong Xi Yi Jie He Za Zhi 35 (12), 1442-1447.

Conflict of Interest: The authors declare that the research was conducted in the absence of any commercial or financial relationships that could be construed as a potential conflict of interest.

Publisher's Note: All claims expressed in this article are solely those of the authors and do not necessarily represent those of their affiliated organizations, or those of the publisher, the editors and the reviewers. Any product that may be evaluated in this article, or claim that may be made by its manufacturer, is not guaranteed or endorsed by the publisher.

Copyright (c) 2021 Zhai, Zou, Wang and Xiao. This is an open-access article distributed under the terms of the Creative Commons Attribution License (CC $B Y)$. The use, distribution or reproduction in other forums is permitted, provided the original author(s) and the copyright owner(s) are credited and that the original publication in this journal is cited, in accordance with accepted academic practice. No use, distribution or reproduction is permitted which does not comply with these terms. 